\title{
Microwave Tumor Ablation of Vertebral Bone Metastasis: A Case Report
}

\author{
Behnam Hosseini ${ }^{1}$, Masoud Hashemi ${ }^{1}$, Mohamad Mehdi Zhand ${ }^{2}$, Babak Alijani ${ }^{3}{ }^{*}$, Reza Jalili-Khoshnood ${ }^{4}$ \\ ${ }^{1}$ MD, Assistant Professor, Pain Medicine Fellowship, Program Director of Pain Fellowship, Department of Anesthesiology and Pain Medicine, Shahid \\ Beheshti University of Medical Sciences, Tehran, Iran \\ ${ }^{2}$ MD, Fellowship of Anesthesiology and Pain Medicine, Shahid Beheshti University of Medical Sciences, Tehran, Iran \\ ${ }^{3}$ MD, Assistant Professor of Neurosurgery, Poursina Hospital, Guilan University of Medical Sciences, Rasht, Guilan, Iran \\ ${ }^{4}$ MD, Assistant Professor of Neurosurgery, Shohada Tajrish Hospital, Shahid Beheshti University of Medical Sciences, Tehran, Iran \\ * Corresponding Author Address: Poursina Hospital, Guilan University of Medical Sciences, Rasht, Guilan, Iran. Tel: +981333368773, \\ Fax: +981333339842. E-mail: babakalij@gmail.com
}

Article Type: Case Report

Received: December 4, 2015, Last revised: December 8, 2015, Accepted: December 21, 2015

\section{Abstract}

Background \& Importance: Thermal ablation by microwave (MWA) has proven efficacy in treatment of primary and metastatic cancers. Soft tissue tumors could be ablated completely using MWA. However, bony tissue metastasis particularly spinal vertebral metastasis ablation has not been reported yet.

Case Presentation: A 50 year old male patient with primary diagnosed colon cancer that had surgical resection was recently diagnosed with metastasis to liver and spinal vertebral bone. He was referred to our pain clinic with severe non-radiating back pain. In MRI Imaging, hyperdensity lesions on body of L5 and T12, and in spinal canal of L1 were identified, indicating metastatic involvement. Under fluoroscopy guidance, the metastatic tumor was ablated by MWA and the generator was powered to 20 Watt/3 minutes in the temperature control mode. Patient had no low back pain afterward.

Conclusion: MWA could be used to ablate vertebral bone metastasis without any complications and with good prognosis.

Keywords: Microwave; Tumor; Ablation; Vertebral Bone; Metastasis

Please cite this paper as: Hosseini B, Hashemi M, Zhand M.M, Alijani B, Jalili-Khoshnood R. Microwave Tumor Ablation of Vertebral Bone Metastasis: A Case Report. Iran. J. Neurosurg. 2015;1(4):25-27.

\section{Introduction}

Thermal ablation by microwave (MWA) has proven its efficacy in treatment of primary and metastatic cancers. It has become standard treatment for selected, small liver, kidney, spleen, and lung tumors that are not suitable for surgical resection (1).

Previous experiences with percutaneous MWA demonstrated that it is a safe and effective modality to devascularize and decrease the size of renal angiomyolipoma (2). Beyer et al. reported a retrospective single-center evaluation of microwave thermoablations of liver tumors in 46 patients (3). Another report of four patients with five pathologically proven splenic metastases demonstrated that all tumors were completely ablated at a single session and no complications occurred (4). Survival analysis demonstrated that two-year overall survival rate in inoperable non-small cell lung cancers (NSCLCs) patients with microwave tumor ablation was higher than that in radiotherapy group (5).

Although soft tissue tumors could be ablated completely using MWA; however, MWA of bony metastasis particularly spinal vertebral metastasis ablation has not been fully studied yet. Recent reports showed that MWA of bone metastases is a well tolerated, safe, and effective treatment of painful refractory spinal metastases (6). However, its efficacy still remains to be determined by medium- and long-term studies (7). Besides, there are other concerns about the efficacy of such treatment modality when used in combination with vertebroplasty. Here in this study we applied MWA for ablating vertebral bones metastasis and then vertebroplasty and long-term follow-up of a patient with spinal bone metastasis.

\section{Case Presentation}

A 50 year old male patient had been diagnosed primarily as colon cancer last year was referred to pain clinic due to severe backache resulted from vertebral metastasis. The patient had excisional resection of colon cancer along with right hemicolectomy last year. He had several courses of chemotherapy after surgery.

After his back pain emergence, metastasis to liver and spinal vertebral bone was diagnosed. He had celiac block for his abdominal pain due to hepatic metastasis a month before that in our pain clinic. Abdominal pain improved thereafter, and pain score was 1 according to the visual analogue scale (VAS=1).

Back pain was a non-radiating low back pain with intensity of 8-9 out of 10.0 on the visual analogue scale. On physical exam, he had spinal vertebral tenderness without radiculopathy on T12, L1 and L5 vertebra. In MRI imaging, hyperdensity lesions on body of L5 and T12 and in spinal canal of L1 were identified, indicating metastatic involvement. The patient whole-body scan 


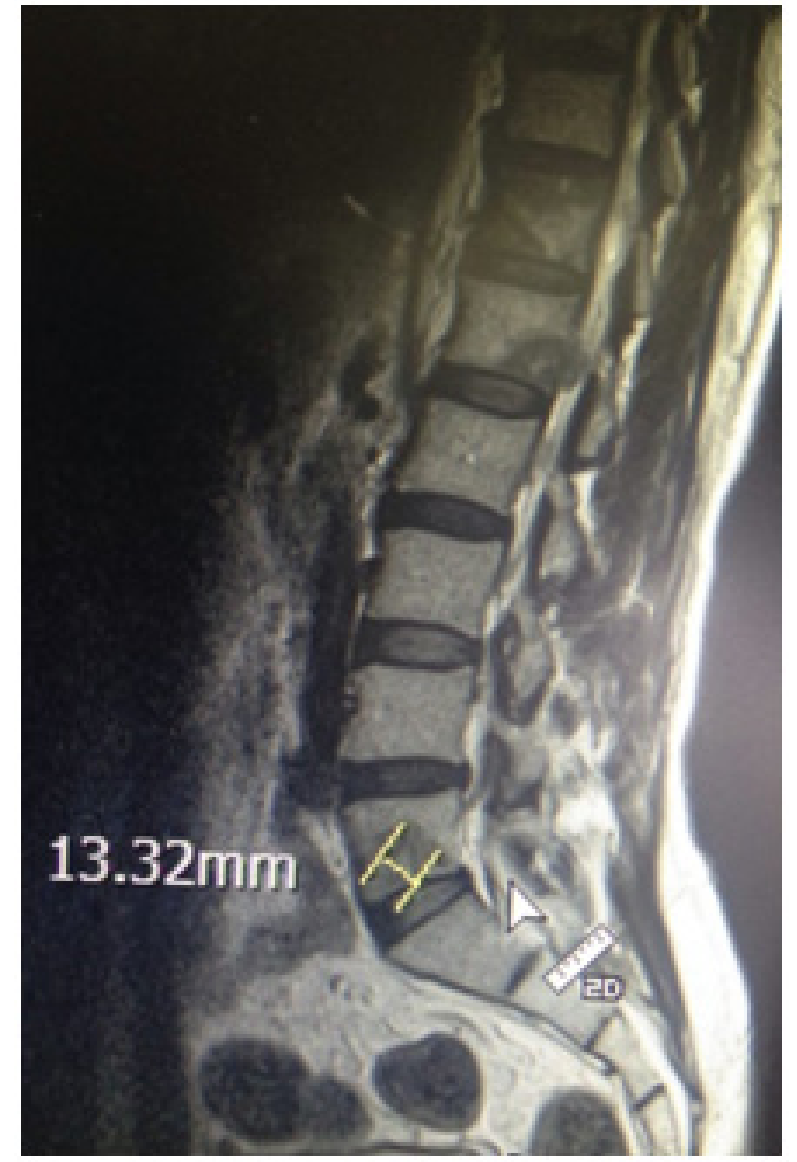

Figure 1. MRI of a Patient Showing Three Metastatic Lesions in Lumbar Vertebra of T12, L1 and L5

confirmed spinal vertebral body metastatic involvement.

\section{Microwave Ablation Protocol}

The patient was informed about the procedure and its possible adverse effects, and a written consent was obtained from the patient and his accompanying person. The patient was put in prone position, and he underwent standard monitoring including ECG, blood pressure, and oxygen saturation. After sedation with $1 \mathrm{mg}$ of midazolam, skin was anesthetized with local anesthetic. Microwave generator (HS Amica-Gen; HS Hospital Service, Rome, Italy) used was capable of producing a maximum power of 80 Watt/15 minutes at the water perfusion-cooled antenna and working at $2.45 \mathrm{GHz}$. In our case, under fluoroscopy guidance, a single, straight microwave antenna (Trochar) 10 $\mathrm{G}$ was placed through transpedicular approach into the body of T12 and L5 vertebra tumor. The generator was powered to $20 \mathrm{Watt} / 3$ minutes in the temperature control mode by cooling in accordance with the manufacturer's recommendations. According to manufacturer's protocol, ablation zone of $24 \mathrm{~mm}$ Length/16 mm Dimension can be reached with this setting (20 Watt $/ 3$ minutes) with an internally cooled antenna within a short ablation time of 3 minutes (9).

Plan was MWA of T12 and L5 tumor ablation under fluoroscopy guide. Tumor ablation was performed, and cement was injected in T12 and L5 (Vertebroplasty). Tumor ablation was not performed on L1 metastasis due to involvement of posterior wall and possibility of leak of cement into epidural space.

The patient low back pain significantly improved $(\mathrm{VAS}=0$ -
1), and he did not complain of any further lumbar vertebral tenderness or low back pain. Pain score was measured at one month intervals, and the follow-up took eight months. Because there were concerns about thermal injury to the spinal nerve roots, we had a full neurologic examination of patient at each follow-up visit. The patient had no sign of any damage to nerves or nerve roots after the MWA procedure during eight month of follow-up visits.
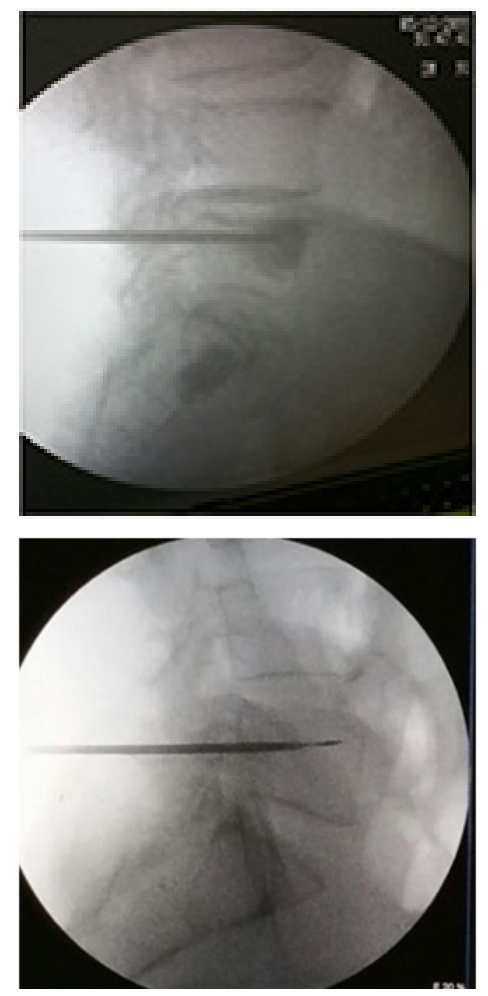

Figure 2. Inserting Microwave Antenna into Metastatic Lesion in Lumbar Vertebra under Fluoroscopy Guide

\section{Discussion}

MWA is a new modality to ablate non-resectable tumors and metastasis. Here, we reported a case of successfull MWA of lumbar vertebral body metastasis which its severe pain in the patient's low back improved. Stättner et al. (2013) performed a retrospective survey on 43 patients who had open MWA with or without resection for colorectal liver metastases (10). They showed that microwave tumor ablation could be a suitable treatment option for small non-resectable colorectal liver metastases. Others depicted that MWA of liver tumors is feasible and appears effective for the treatment of small liver tumors (less than three centimeters) (10).

Our patient was one of the first cases of lumbar vertebral body metastasis that was successfully ablated by MWA. Bony vertebral body metastasis could be painful, and it caused decreased life quality and disordered ambulation for the patient. MWA offers several advantages over radiofrequency ablation (RFA), including faster heating over a larger volume, simultaneous use of multiple applicators and no requirement for ground pads (11). The main differences between MWA and RFA are maximum temperature in the two techniques. In RFA, temperature rises only up to $90^{\circ} \mathrm{C}$, while temperature goes up to $180^{\circ} \mathrm{C}$ in MWA. Besides, RFA depends on active point which limits its effectiveness, while MWA does not depend on active point that enhances its effectiveness in this case. Therefore, 
using MWA instead of RFA, active heating increases and may induce cellular death more uniformly in the target area (12). MWA device may allow more uniform ablations in a shorter period of time compared with currently RFA system. Altogether, more recurrence rate is observed in RFA than MWA.

Although there are concerns about thermal injury to the spinal nerve roots, we did not observe any sign of neurological damage to our patient in the neurologic exam during 8 month of follow-up. Besides, there is no report on such thermal injury in previous researches. Nevertheless, further neurologic studies or lab tests should be carried out in the future to confirm the safety of MWA to the spinal nerve roots during spinal bone MWA. There is a paucity of systematically obtained clinical data to give a perspective to the actual magnitude of the risks and benefits associated with ablation of spinal vertebral body metastasis.

\section{Conclusion}

In conclusion, MWA could be used to ablate vertebral bone metastasis without any complications and with good prognosis.

\section{Funding}

None.

\section{Conflicts of Interest}

The authors declare that they have no conflicts of interest.

\section{References}

1. Poon RT-P, Fan S-T, Tsang FH-F, Wong J. Locoregional therapies for hepatocellular carcinoma: a critical review from the surgeon's perspective. Annals of surgery. 2002;235(4):466-86.

2. Cristescu M, Abel EJ, Wells S, Ziemlewicz TJ, Hedican SP, Lubner $\mathrm{MG}$, et al. Percutaneous Microwave Ablation of Renal Angiomyolipomas. Cardiovascular and interventional radiology. 2015:1-8.

3. Beyer L, Pregler B, Niessen C, Dollinger M, Graf B, Müller M, et al. Robotassisted microwave thermoablation of liver tumors: a single-center experience. International journal of computer assisted radiology and surgery. 2015:1-7.

4. Yu J, Liang P, Yu X, Wang Y, Gao Y. Ultrasound-guided percutaneous microwave ablation of splenic metastasis: report of four cases and literature review. International Journal of Hyperthermia. 2011;27(5):517-22.

5. Xu X, Ye X, Liu G, Zhang T. Targeted percutaneous microwave ablation at the pulmonary lesion combined with mediastinal radiotherapy with or without concurrent chemotherapy in locally advanced non-small cell lung cancer evaluation in a randomized comparison study. Medical Oncology. 2015;32(9):1-8

6. Kastler A, Alnassan H, Aubry S, Kastler B. Microwave thermal ablation of spinal metastatic bone tumors. Journal of Vascular and Interventional Radiology. 2014;25(9):1470-5.

7. Pusceddu C, Sotgia B, Fele RM, Melis L. Treatment of bone metastases with microwave thermal ablation. Journal of Vascular and Interventional Radiology. 2013;24(2):229-33.

8. Flier JS, Underhill LH, Klibanski A, Zervas NT. Diagnosis and management of hormone-secreting pituitary adenomas. New England Journal of Medicine. 1991;324(12):822-31.

9. Goldberg S, Solbiati L, Hahn P, Cosman E, Conrad J, Fogle R, et al. Largevolume tissue ablation with radio frequency by using a clustered, internally cooled electrode technique: laboratory and clinical experience in liver metastases. Radiology. 1998;209(2):371-9.

10. Stättner S, Jones R, Yip V, Buchanan K, Poston G, Malik H, et al. Microwave ablation with or without resection for colorectal liver metastases. European Journal of Surgical Oncology (EJSO). 2013;39(8):844-9.

11. Brace CL. Microwave ablation technology: what every user should know. Current problems in diagnostic radiology. 2009;38(2):61-7.

12. Wolf FJ, Aswad B, Ng T, Dupuy DE. Intraoperative microwave ablation of pulmonary malignancies with tumor permittivity feedback control: ablation and resection study in 10 consecutive patients. Radiology. 2012;262(1):353-60.

\section{Comments}

We read the paper entitled microwave tumor ablation of vertebral bone metastasis: A case report with great interest. Author/s introduced a 50-year-old male with colon cancer and metastasis of liver and vertebral bone. Patient had severe low back pain. There were at least three vertebra involvements in T12, L1, and L5. L1 vertebral body and posterior wall was involved and tumor had progressed to the epidural space and compressed conus and cauda equine. In T12 and L5, vertebral bodies were involved. Author/s showed the significant pain decrease following microwave thermal ablation and vertebroplasty of T12 and L5 without any mentioned treatment on L1. Visual Analogue Scale (VAS) decreased from $9 / 10$ to $0-1 / 10$; and pain free period persisted from first to eighth month during every month follow-ups. Author/s did not write anything about other additional radiotherapy or chemotherapy for liver and bone metastasis. We do not know when pain started to decrease and if the pain decrease was gradual or not. Author/s did not mention if the L1 metastasis produced any neurologic deficit during eight month follow-up and if the patient died after eight months. Literature supports the value of microwave ablation in decreasing bone metastatic pain. However, the standard treatment includes additional local radiotherapy (1-4).

Vafa Rahimi-Movaghar, MD, Professor of Neurosurgery, Research Vice President of Sina Trauma and Surgery Research Center.

Department of Neurosurgery, Shariati Hospital, Tehran University of Medical Sciences, Tehran, Iran.

AOSpine Research Officer of Middle East, Director of National Spinal Cord Injury in Iran, Research Center for Neural Repair, University of Tehran

\section{References}

1. Palussière J, Buy X, Fonck M. Percutaneous ablation of metastases: where are we and new techniques. Bull Cancer. 2013;100(4):373-9. [French]

2. Carrafiello G, Dionigi G, Boni L, Mangini M, Ierardi AM, Piacentino F, Di Massa A, Nocchi Cardim L, Biondi A, Fugazzola C. Current role of interventions in metastatic kidney tumors: single center experience. Updates Surg. 2011;63(4):259-69.

3. Carrafiello $\mathrm{G}$, Laganà D, Pellegrino $\mathrm{C}$, Fontana $\mathrm{F}$, Mangini $\mathrm{M}$, Nicotera $\mathrm{P}$, Petullà $\mathrm{M}$, Bracchi E, Genovese E, Cuffari S, Fugazzola C. Percutaneous imaging-guided ablation therapies in the treatment of symptomatic bone metastases: preliminary experience. Radiol Med. 2009;114(4):608-25. [English, Italian]

4. Simon CJ, Dupuy DE. Percutaneous minimally invasive therapies in the treatment of bone tumors: thermal ablation. Semin Musculoskelet Radiol. 2006;10(2):137-44. 